\title{
Aryl hydrocarbon receptor nuclear translocator promotes the proliferation and invasion of clear cell renal cell carcinoma cells potentially by affecting the glycolytic pathway
}

\author{
YUXIAO ZHAO ${ }^{1}$, FENG HAN $^{2}$, XUFENG ZHANG $^{3}$, CHENGJUN ZHOU $^{4}$ and DEQIANG HUANG ${ }^{5}$ \\ ${ }^{1}$ Queen Mary School, Nanchang University, Nanchang, Jiangxi 330031; ${ }^{2}$ Department of Endocrinology, \\ The People's Hospital of Zhangqiu Area, Jinan, Shandong 250200; Departments of ${ }^{3}$ Kidney Transplantation and ${ }^{4}$ Pathology, \\ The Second Hospital of Shandong University, Jinan, Shandong 250033; ${ }^{5}$ Department of Gastroenterology, \\ Research Institute of Digestive Diseases, The First Affiliated Hospital of Nanchang University, \\ Nanchang, Jiangxi 330006, P.R. China
}

Received February 15, 2020; Accepted June 29, 2020

DOI: 10.3892/ol.2020.11917

\begin{abstract}
Aryl hydrocarbon receptor nuclear translocator (ARNT) is a transcription factor that has been reported to play a vital role in regulating glycolysis, angiogenesis and apoptosis. Recently, ARNT has been reported to a play role in pancreatic-islet function in type 2 diabetes. However, the role of ARNT in kidney cancer has not yet been investigated. In the present study, ARNT expression was detected in tissues from patients with renal cell carcinoma (RCC) and in RCC cell lines. Oncomine, The Cancer Genome Atlas and cBioPortal were used to investigate the roles of ARNT in RCC. Cell migration and invasion assays were used to explore the molecular mechanisms involved. It was found that ARNT protein expression was elevated both in tissues from patients with clear cell RCC (ccRCC) and in different RCC cell lines. ARNT disruption using siRNA knockdown inhibited the migratory abilities and cell proliferation, potentially by altering the glycolysis pathway in vitro, as evidenced by decreased M2 type acetone kinase, 6-phosphofructo-2-kinase/fructose-2,6-bisphosphatase 3 and hexokinase 2 expression. Taken together, the findings in the present study revealed a novel function of ARNT in ccRCC and indicated that ARNT promotes the proliferation and invasion of ccRCC, possibly through changes to the glycolytic pathway.
\end{abstract}

Correspondence to: Dr Deqiang Huang, Department of Gastroenterology, Research Institute of Digestive Diseases, The First Affiliated Hospital of Nanchang University, 17 Yongwaizheng Street, Nanchang, Jiangxi 330006, P.R. China

E-mail: hdq0515@ncu.edu.cn

Key words: aryl hydrocarbon receptor nuclear translocator, renal cell carcinoma, kidney cancer, glycolysis, bioinformatics

\section{Introduction}

Renal cell carcinoma (RCC) accounts for $2 \%$ of adult malignancies worldwide and for $80-85 \%$ of all malignant kidney tumors, according to data from $2013(1,2)$. Clear cell carcinoma is the most common pathological subtype of RCC and is highly resistant to both chemotherapy and radiation therapy (3). Metabolic reprogramming is of the utmost importance to oncogenesis, but the generation and effects of this profound process remain unclear. Compared with normal cells, glycolysis is enhanced and the mitochondrial oxidative phosphorylation capacity is reduced in various cancer cell types, such as in malignant glioma, leukemia and colon cancer cell lines $(4,5)$. It has been reported that inhibiting the expression or activity of key glycolytic enzymes can effectively inhibit tumor cell proliferation and even kill tumor cells. Such enzymes include lactate dehydrogenase (LDHA), hexokinase 2 (HK2), phosphofructokinase (PFK) and M2 type acetone kinase (PKM2) (6,7). Given the increasing incidence of this cancer and its lack of effective therapeutic targets, there is an urgent requirement to identify the potential mechanisms by which clear cell RCC (ccRCC) behavior is regulated.

Hypoxia has long been implicated in genetic instability and tumor progression. Hypoxia inducible factors (HIFs) are central players in cellular hypoxia adaptation, and the HIF-1 signaling pathway is critical to tumor development and progression $(8,9)$. HIF-1 [also known as aryl hydrocarbon receptor nuclear translocator (ARNT)] is a heterodimeric transcription factor composed of HIF-1 $\alpha$ and HIF-1 $\beta$ (10). ARNT is a member of the basic helix-loop-helix PER-ARNT-SIM family (11); it is a central player in two cellular signaling pathways, namely the aryl hydrocarbon receptor and HIF pathways $(12,13)$. Previous studies have indicated that the expression of ARNT is decreased in pancreatic islets from humans with type 2 diabetes (14-16). ARNT expression is critical to normal angiogenesis and glycolysis, and the prevention of apoptosis (17). However, no evidence has been reported concerning the expression and potential functions of ARNT in ccRCC. 
The present study aimed to investigate the role of ARNT and the possible mechanisms by which it influences the glycolytic pathway in ccRCC using cBioPortal analysis and in vitro experiments, in order to provide evidence regarding the role of ARNT in ccRCC.

\section{Materials and methods}

Dataset collection and samples from patients with ccRCC. A total of 58 patients with ccRCC who had never previously been treated with radiotherapy or chemotherapy were enrolled for the study. According to ARNT expression, patients were divided into three groups, and their clinical characteristics are shown in Table I. Tumor tissues were taken from the surgically resected tissues of patients with ccRCC treated at The Second Hospital of Shandong University (Jinan, China) between April 2018 and April 2019. All patients provided written informed consent. Furthermore, this study was approved by the Human Research Ethics Committee of The Second Hospital of Shandong University.

Oncomine (http://www.oncomine.org), an online microarray database, was used to analyze differences in mRNA expression for ARNT and key enzymes involved in the glycolysis pathway [LDHA, HK2, PKM2 and 6-phosphofructo-2-kinase/fructose-2,6-bisphosphatase 3 (PFKFB3)] between tumor and normal tissues from patients with ccRCC. Genes were considered to be significantly differentially expressed using $\mathrm{P}<0.05$ and $|\log \mathrm{FC}|>2$.

Preprocessed level 3 RNA-seq data were downloaded from The Cancer Genome Atlas (TCGA) data portal (https://portal.gdc.cancer.gov). For TCGA data, the edgeR package version 3.30.3 (https://bioconductor.org/packages/edgeR/) was used for differentially expressed gene (DEG) screening. $\mathrm{P}<0.05$ and $\mid \mathrm{LogFCl}>2$ were chosen as the cut-off criteria.

Alterations in the ARNT gene occurring in ccRCC were investigated using cBioPortal (http://www.cbioportal.org) to generate a network of the interactions between ARNT and its neighboring genes.

Immunohistochemistry. Tumor tissues from patients with ccRCC were fixed in $4 \%$ paraformaldehyde for $24-48 \mathrm{~h}$ at room temperature, dehydrated using 70,80 and $95 \%$ alcohol (45 min each), followed by 3 washes using 100\% alcohol ( $1 \mathrm{~h}$ each), and cleared using 2 washes of xylene ( $1 \mathrm{~h}$ each), and then embedded in paraffin. Paraffin-embedded tissues were cut into 4- $\mu \mathrm{m}$-thick sections. Subsequently, the sections were stained using the Mouse two-step test kit (Mouse reinforced Polymer test System; cat. no. PV-9002; OriGene Technologies, Inc.) according to the manufacturer's protocol. Briefly, to inactivate endogenous peroxidase, an appropriate amount of endogenous peroxidase blocker (included in the Mouse two-step test kit) was added at room temperature for $10 \mathrm{~min}$, while to block non-specific antibody binding, the slides were incubated with $5 \%$ bovine serum albumin (cat. no. 0332; GBCBIO Technologies, Inc.) for $30 \mathrm{~min}$ at room temperature. Subsequently, the slides were incubated with an anti-ARNT primary antibody $\left(1: 200\right.$; cat. no. ab2771; Abcam) at $4{ }^{\circ} \mathrm{C}$ overnight. The slides were washed 3 times ( 3 min each) in PBS buffer (Gibco; Thermo Fisher Scientific, Inc.). Next, the
Table I. Patient clinical characteristics.

\begin{tabular}{lccc}
\hline Characteristic & $\begin{array}{c}\text { Group 1 } \\
(\mathrm{n}=12)\end{array}$ & $\begin{array}{c}\text { Group 2 } \\
(\mathrm{n}=17)\end{array}$ & $\begin{array}{c}\text { Group 3 } \\
(\mathrm{n}=29)\end{array}$ \\
\hline ARNT expression & Weak & Moderate & Strong \\
Male/Female, $\mathrm{n}$ & $5 / 7$ & $11 / 6$ & $20 / 9$ \\
$\begin{array}{l}\text { Mean age } \\
\text { (range), years }\end{array}$ & $56.8(39-76)$ & $55.0(38-70)$ & $58.2(35-82)$ \\
\hline
\end{tabular}

Enhance enzyme-labeled secondary antibody (goat anti-mouse $\mathrm{IgG}$ ) from the kit was applied to the slides and incubated in a humidified chamber at room temperature for $1 \mathrm{~h}$. Images were taken using a light microscope (magnification, x200). Nuclei were counterstained using a hematoxylin counterstain reagent (Roche Diagnostics) for 1-2 min at room temperature. Scoring was based on the color intensity: 0 , no staining; 1 , light yellow; 2 , yellow-brownish; and 3, brown. Scoring of the percentage of positive cells was performed as follows: $0,1-10 \% ; 1,11-25 \%$; $2,26-50 \% ; 3,51-75 \%$; and 4, 76-100\%. Finally, the two scores were multiplied to obtain the final score: 0 , negative; $1-4$, weak; 5-8, moderate; and 9-12, strong expression.

Cell culture and transfection. The normal human kidney HK-2 cell line and the RCC cell lines A498, OS-RC-2 and 786-O were purchased from The Cell Bank of Type Culture Collection of the Chinese Academy of Science. The HK-2 cell line was used as a normal control of RCC cell lines. A498, OS-RC-2 and 786-O cells were used to study the function of ARNT in RCC cell lines. In a humidified $5 \% \mathrm{CO}_{2}$ environment at $37^{\circ} \mathrm{C}$, $\mathrm{HK}-2$ and OS-RC-2 cells were cultured in DMEM supplemented with 10\% FBS (both Gibco; Thermo Fisher Scientific, Inc.). A498 and 786-O were cultured in RPMI 1640 (Gibco; Thermo Fisher Scientific, Inc.) supplemented with $10 \%$ FBS. To generate ARNT-overexpressing stable cell populations (OV-ARNT), OS-RC-2 cells were infected with an empty vector control and an ARNT lentivirus vector, which were constructed by Shanghai GeneChem Co., Ltd. OS-RC-2 cells were seeded until they reached $60-80 \%$ confluency the following day. After the cells were attached to the walls, they were infected with ARNT lentivirus or control lentivirus for $16 \mathrm{~h}$, the medium was replaced with fresh DMEM supplemented with $10 \%$ FBS and subsequent experiments were performed after $48 \mathrm{~h}$. The virus was used at a multiplicity of infection of 10 to infect OS-RC-2. The efficiency of infection was assessed via western blot analysis. To generate ARNT-knockdown stable cell populations (si-ARNT), three siRNAs to ARNT and a negative control (NC) siRNA were ordered from Guangzhou RiboBio Corporation Co., Ltd. A498 cells were seeded for $24 \mathrm{~h}$ until they reached $60-80 \%$ confluency, and were then transfected with $20 \mu \mathrm{M}$ siRNAs using Oligofectamine ${ }^{\mathrm{TM}}$ reagent (Invitrogen; Thermo Fisher Scientific, Inc.) according to the manufacturer's protocol. Subsequent experiments were conducted $6 \mathrm{~h}$ after transfection. The sequences used for the knockdown experiments were as follows: NC sense, 5'-UUCUCCGAACGU GUCACGUTT-3' and antisense, 5'-ACGUGACACGUUCGG AGAATT-3'; ARNT-homo-926 (si-ARNT-homo-1) sense, 5'-GGCUCA AGGAGAUCGUUUATT-3' and antisense, 
A

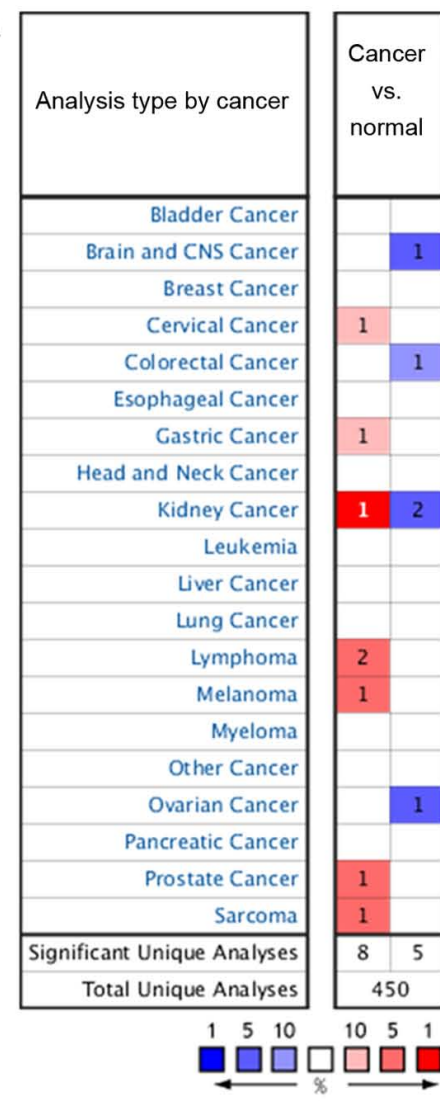

B
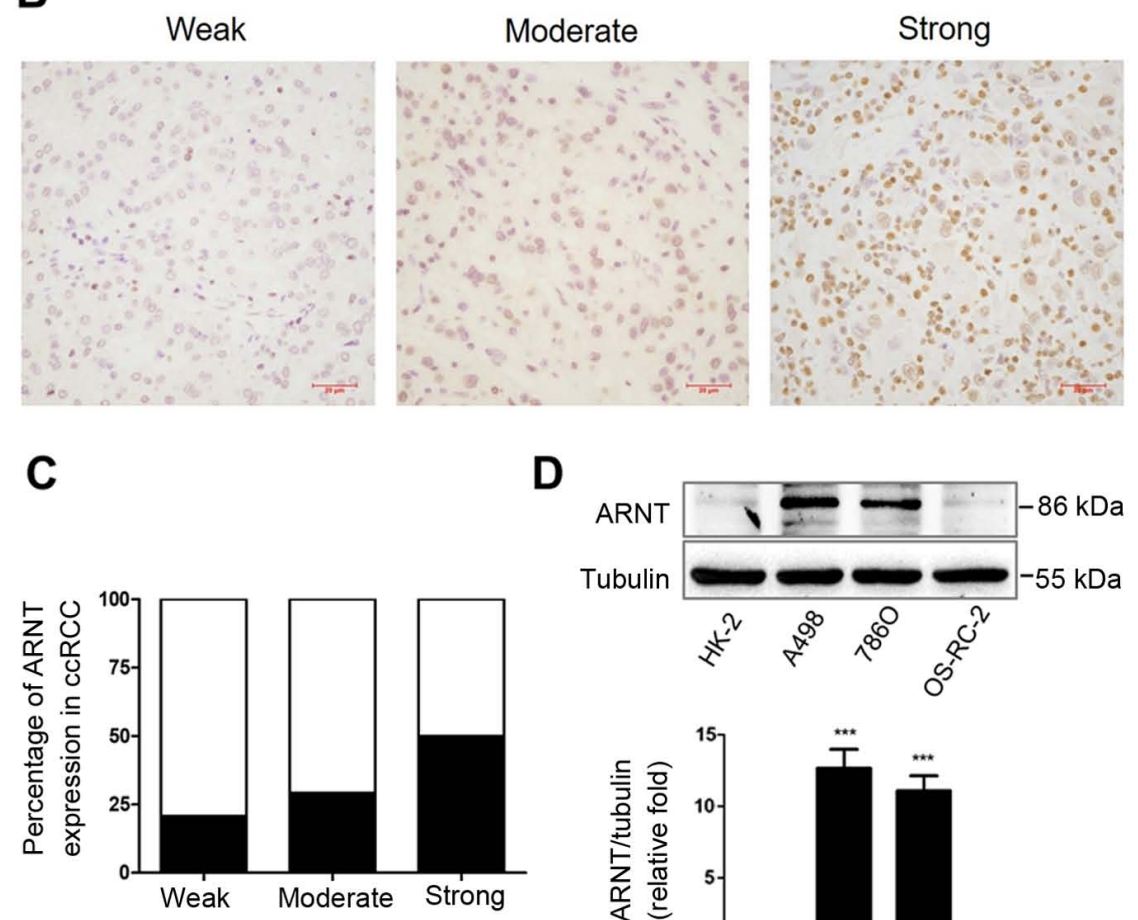

Figure 1. ARNT expression profiles in tissues and RCC cell lines. (A) Summary of ARNT expression in different cancer types. (B) The immunohistochemical staining analyses of ARNT in tumor tissues of patients with ccRCC (magnification, x200; scale bar, $20 \mu \mathrm{m}$ ). (C) Percentage quantification of immunohistochemical staining. The sum of the black and white areas indicate the total, and the black area indicates the percentage occupied by each group. (D) Protein levels of ARNT in RCC cell lines. ${ }^{* * *} \mathrm{P}<0.001$ vs. HK-2. ARNT, aryl hydrocarbon receptor nuclear translocator; ccRCC, clear cell renal cell carcinoma.

5'-UAAACGAUCUCCUUGAGCCTT-3'; ARNT-homo-1442 (si-ARNT-homo-2) sense, 5'-CGGUCUAAGAACCAAGAA UTT-3' and antisense, 5'-AUUCUUGGUUCUUAGACC GTT-3'; and ARNT-homo-1942 (si-ARNT-homo-3) sense, 5'-GGCAGAGAAUUUCAGGAAUTT-3' and antisense, 5'-AUUCCUGAAAUUCUCUGCCTT-3'.

Cell migration and invasion assays. Cell migration and invasion assays were performed as previously described (18). Briefly, $5 \times 10^{5}$ cells were placed in 6 -well plates. The migration rate of OS-RC-2 and A498 cells pre-transfected with ARNT or control was assessed by wound-healing assays, with $1 \%$ FBS used in the culture medium. Next, photomicrographs were captured of OS-RC-2 and A498 cells under a light microscope (Leica DFC300FX; Leica Microsystems, Inc.; magnification, $\mathrm{x} 40$ ) at 0 and $24 \mathrm{~h}$. Transwell invasion assays were performed in OS-RC-2 and A498 cells pre-transfected with ARNT or control $\left(3 \times 10^{4}\right.$ cells/well). Cells were placed in an 8-mm Transwell cell culture chamber (Corning Inc.) FBS-free DMEM was added in the upper chamber, while DMEM with $10 \%$ FBS was used in the lower chamber. The cells and Matrigel (Becton, Dickinson and Company) were incubated in a $37^{\circ} \mathrm{C}$ incubator for $36 \mathrm{~h}$. After $36 \mathrm{~h}$, the cells were fixed with methanol for $30 \mathrm{~min}$ at room temperature and stained using crystal violet solution (1X PBS, $0.05 \%$ $\mathrm{w} / \mathrm{v}$ crystal violet, $1 \%$ formaldehyde and $1 \%$ methanol) for $30 \mathrm{~min}$ at room temperature. Cells in the upper chamber were removed using a cotton swab. The migrated cells were observed under a light microscope (Olympus Corporation; magnification, $x 100$ ) and counted manually from five randomly selected fields.

Cell proliferation assays. To investigate the sensitivity to sorafenib (LC Laboratories), the Cell Counting Kit-8 (CCK-8; Beyotime Institute of Biotechnology) assay was used to detect the cell survival rate, according to the manufacturer's protocol. Cells were treated with different concentrations of sorafenib $(5,10$ or $20 \mu \mathrm{M})$ for $48 \mathrm{~h}$ at $37^{\circ} \mathrm{C}$. DMSO group served as a control. Briefly, $5 \times 10^{3}$ OS-RC-2 or A498 cells were seeded in a 96-well plate and cell proliferation was evaluated after $48 \mathrm{~h}$ of sorafenib treatment. Next, $10 \mu \mathrm{l}$ WST-1 reagent was added per well and the cultures were incubated for $2 \mathrm{~h}$, after which the absorbance was measured at $450 \mathrm{~nm}$ using a microplate reader (BioTek Instruments Inc.).

Western blot analysis and antibodies. Western blotting was performed as previously described (19). Briefly, cultured cells (HK-2, A498, OS-RC-2 and 786-O) were lysed using radioimmunoprecipitation buffer (cat. no. P0013B; Beyotime Institute of Biotechnology) and centrifuged at 13,000 x $\mathrm{g}$ for $20 \mathrm{~min}$ at $4^{\circ} \mathrm{C}$. Protein concentrations of the extracts were determined using a BCA protein assay kit (cat. no. 23227; Pierce; Thermo Fisher Scientific, Inc.). Soluble lysates (20 $\mu \mathrm{g} /$ lane) were subjected to $10 \%$ SDS-PAGE and transferred 
A
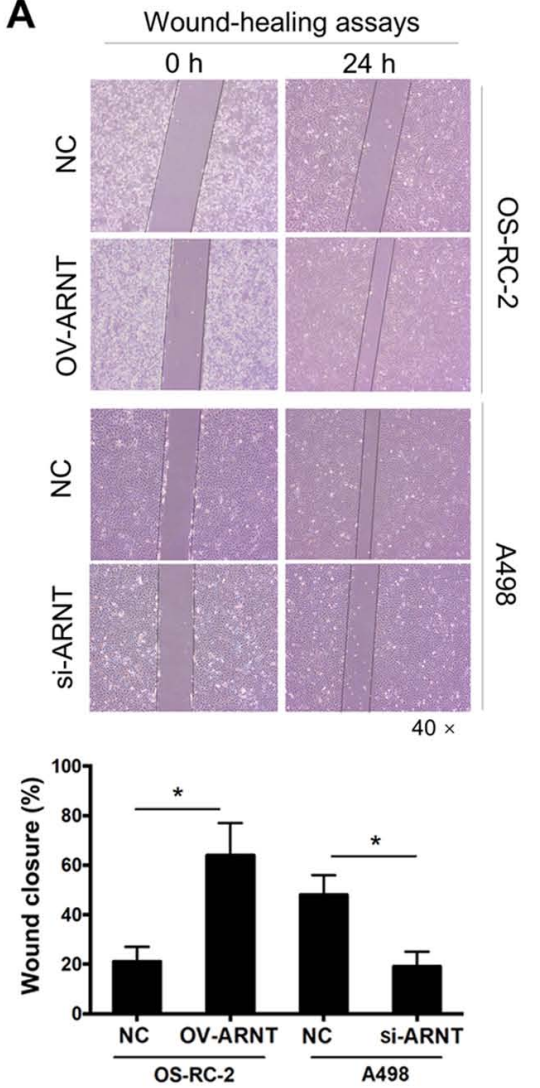

B
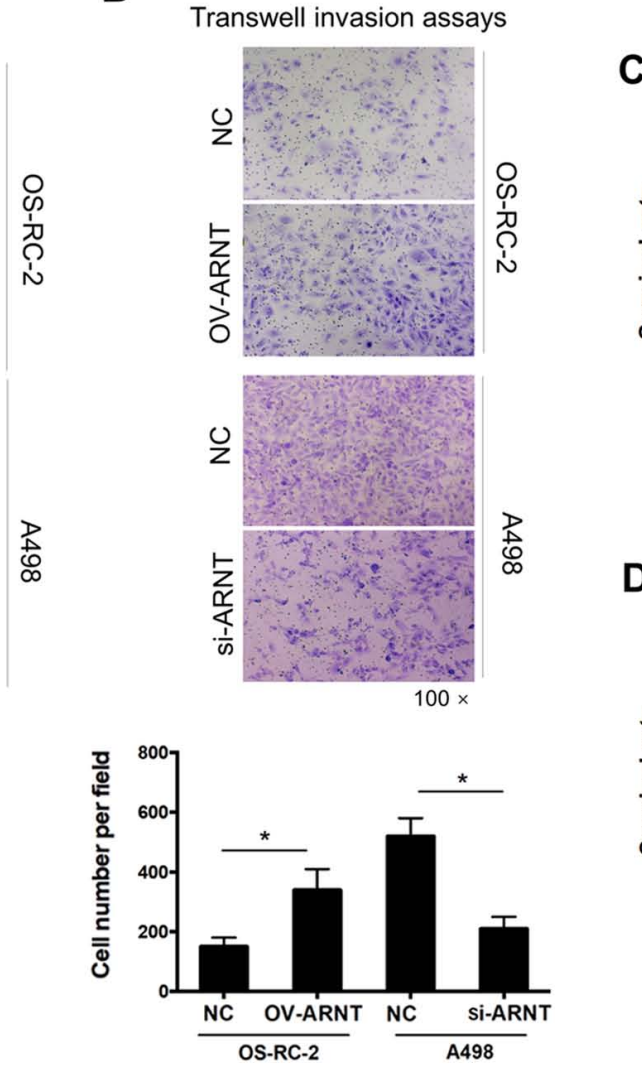

C

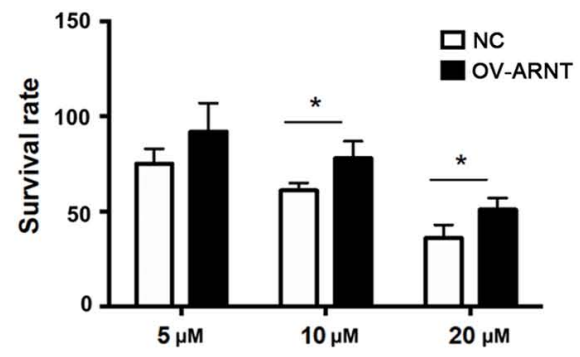

D

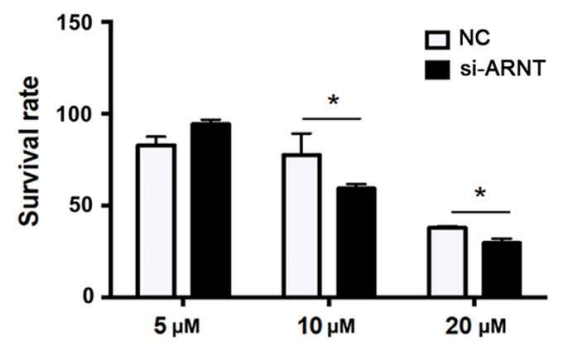

Figure 2. Aberrant ARNT expression leads to changes in the cellular behavior of RCC cells. OS-RC-2 cells were transfected with ARNT to overexpress ARNT, and RCC cells A498 were infected with a lentiviral vector carrying siRNA to silence endogenous ARNT. (A) The migration rate of OS-RC-2 and A498 cells pretreated for $24 \mathrm{~h}$ was observed through wound-healing assays. Magnification, $\mathrm{x} 40$. (B) Transwell invasion assays were conducted to observe the invasive cells in OS-RC-2 and A498 cell lines. Magnification, x100. Cell Counting Kit-8 assays in (C) OS-RC-2-OV-ARNT and (D) A498-si-ARNT cells were treated with different doses of sorafenib as indicated. Cell proliferation was examined $48 \mathrm{~h}$ after treatment using the CCK-8 assay. $\mathrm{P}<0.05$. ARNT, aryl hydrocarbon receptor nuclear translocator; ccRCC, clear cell renal cell carcinoma; si, small interfering; NC, negative control; OV, overexpression.

to nitrocellulose membranes (cat. no. 10402495; Whatman plc; Cytiva). Membranes were incubated with primary antibodies overnight at $4^{\circ} \mathrm{C}$ and then with HRP-conjugated secondary antibodies for $1 \mathrm{~h}$ at room temperature. ECL Prime Western Blotting Detection Reagent (cat. no. RPN2232; GE Healthcare) was used to visualize protein bands via Image Quant LAS4000 Imaging Systems (GE Healthcare). Relative protein contents were quantified using the Quantity One software (v462 version. Bio-Rad Laboratories, Inc.). Proteins were assessed using the following primary antibodies (diluted in 5\% BSA): ARNT (1:4,000; cat. no. ab2771), LDHA (1:1,000; cat. no., ab101562), HK2 (1:500; cat. no. ab104836), PKM2 (1:1,000; cat. no. ab85555), PFKFB3 (1:1,000; cat. no. ab181861), tubulin (1:500, cat. no. ab6046) and $\beta$-actin (1:200; cat. no. ab115777) (all Abcam). HRP-linked secondary antibodies included anti-rabbit IgG (cat. no. 7074) and anti-mouse $\operatorname{IgG}$ (cat. no. 7076) (both diluted 1:1,000 in 5\% BSA; Cell Signaling Technology, Inc.).

Statistical analysis. All data were analyzed using SPSS version 18.0 (SPSS, Inc.). Data are presented as the mean \pm standard error. Statistical differences were calculated by two-tailed unpaired Student's t-test or one-way ANOVA and Tukey's post hoc test. $\mathrm{P}<0.05$ was used to indicate a statistically significant difference.

\section{Results}

Expression profiles of ARNT in patients with ccRCC and in different RCC cell lines. ARNT is a transcription factor that plays a critical role in the response to environmental stresses, such as dioxin exposure and hypoxia (11). However, few studies have explored its functions, particularly its physiological role in ccRCC. Therefore, the present study examined the expression profiles of ARNT using the Oncomine database. ARNT expression was found to be significantly upregulated in 8 analyses and downregulated in 5 analyses in different cancer types (Fig. 1A). Next, to validate the expression profiles of ARNT, immunohistochemical staining was performed to examine ARNT expression in tumor tissues from 58 patients with ccRCC (Fig. 1B). Notably, nearly $50 \%$ of the clinical samples showed that ARNT was strongly upregulated (Fig. 1C). Furthermore, varying levels of ARNT expression were detected in different RCC cell lines in vitro. A498 and 786-O RCC cell lines showed significantly elevated expression of ARNT compared with the human renal tubular epithelial cell line (HK-2), while there was no significant difference in expression in the OS-RC-2 cell line (Fig. 1D).

ARNT promotes the proliferation, migration and invasion of human ccRCC cells. To investigate the roles of ARNT in the biological behavior of ccRCC cells, overexpression 
A

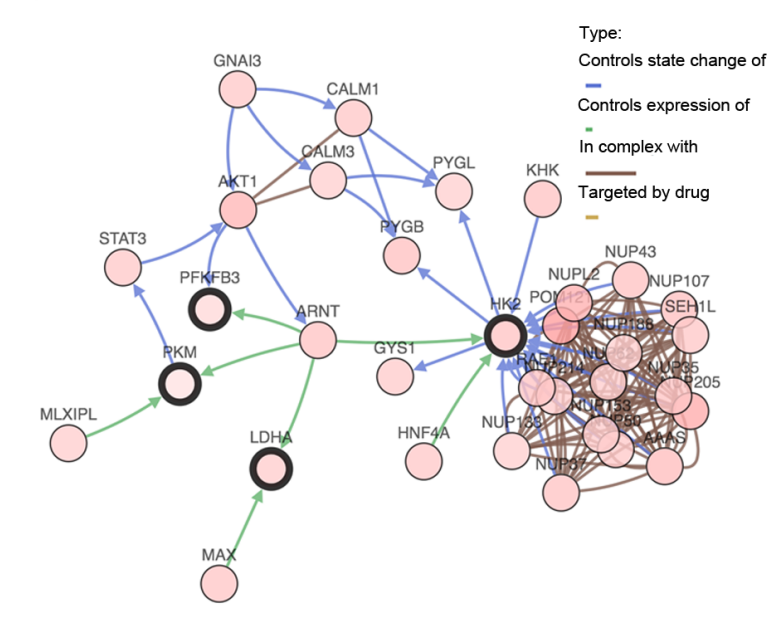

B
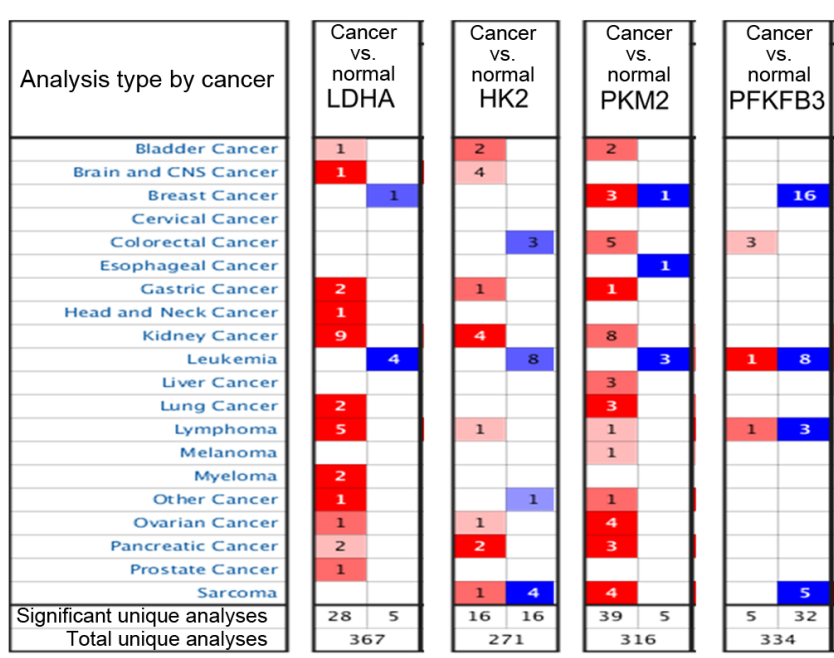

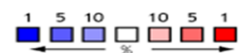

C
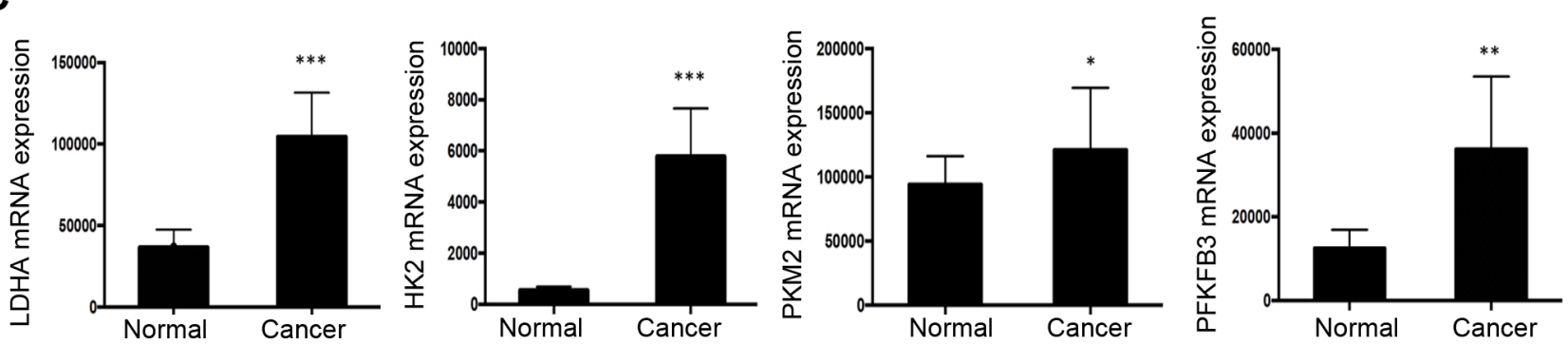

Figure 3. Network of ARNT and altered neighboring genes. (A) Interaction of ARNT with other genes was analyzed in the cBioPortal database. (B) Summary of LDHA, HK2, PKM2 and PFKFB3 expression in different cancer types. (C) The clinical significance of glycolysis pathway protein (LDHA, HK2, PKM2 and PFKFB3) expression in ccRCC, based on The Cancer Genome Atlas data, was investigated. ${ }^{*} \mathrm{P}<0.05,{ }^{* *} \mathrm{P}<0.01$ and ${ }^{* * * *} \mathrm{P}<0.001$ vs. normal. ARNT, aryl hydrocarbon receptor nuclear translocator; PFKFB3, 6-phosphofructo-2-kinase/fructose-2,6-bisphosphatase 3; LDHA, lactate dehydrogenase; HK2, hexokinase; PFK, phosphofructokinase; PKM2, M2 type acetone kinase.

and knockdown experiments were performed. In Fig. 1D, the A498 and 786-O RCC cell lines showed significantly upregulated ARNT expression compared with the human renal tubular epithelial cell line (HK-2), while there was no significant difference in ARNT expression in the OS-RC-2 cell line. Therefore, the OS-RC-2 cell line was selected for ARNT overexpression, and A498 cells were chosen for ARNT-knockdown, which involved transfection of ARNT into OS-RC-2 cells and silencing of endogenous ARNT expression in A498 cells using a lentiviral vector. Compared with silencing ARNT in A498 cells, overexpression of ARNT in OS-RC-2 cells resulted in stronger migratory abilities and accelerated cell proliferation. The wound-healing assay showed greater wound closure in the OS-RC-2 OV-ARNT group compared with that in the control group at $24 \mathrm{~h}$. By contrast, less wound closure was noted in the A498-siRNA-ARNT group compared with that in the control group (Fig. 2A). Significantly higher migration rates were observed in OS-RC-2 OV-ARNT cells, as revealed by Transwell migration assays (Fig. 2B). Sorafenib is the first oral multi-kinase inhibitor that targets Raf and affects tumor cell proliferation and tumor angiogenesis (20). It has been reported that sorafenib treatment prolongs survival rates in patients with ccRCC in whom previous therapy has failed (21). To investigate whether the increased sensitivity to Sorafenib in ccRCC is associated with ARNT expression, the cell survival rate was examined $48 \mathrm{~h}$ after treatment with sorafenib using the CCK- 8 assay. After ARNT overexpression, the sensitivity of OS-RC-2 OV-ARNT cells to sorafenib was decreased and the cell survival rate was increased compared with that of OS-RC-2 NC cells at 10 and $20 \mu \mathrm{M}$ (Fig. 2C). By contrast, the survival rate of the A498-siRNA-ARNT cells was adversely affected compared with that of the NC (Fig. 2D). These results suggest ARNT may be a target of sorafenib, the effects of which could improve the treatment of drug-resistant ccRCC.

ARNT is involved in the glycolysis pathway. Next, the possible mechanism influencing ARNT function was investigated. A network of the interactions of ARNT with several frequently altered neighboring genes was drawn using cBioPortal (Fig. 3A). The network reflected a clear interaction between ARNT and several glycolytic genes, including LDHA, HK2, PKM2 and PFKFB3. Moreover, in the Oncomine database, key glycolysis pathway enzymes (LDHA, HK2 and PKM2) were significantly upregulated in kidney cancer (Fig. 3B). Additionally, based on TCGA dataset, a set of glycolysis pathway enzymes, including LDHA, HK2, PKM2 and PFKFB3, were identified from the DEGs, all of which were significantly upregulated (Fig. 3C). These results suggest that ARNT may contribute to the cancer program through effects on the glycolysis pathway. 
A
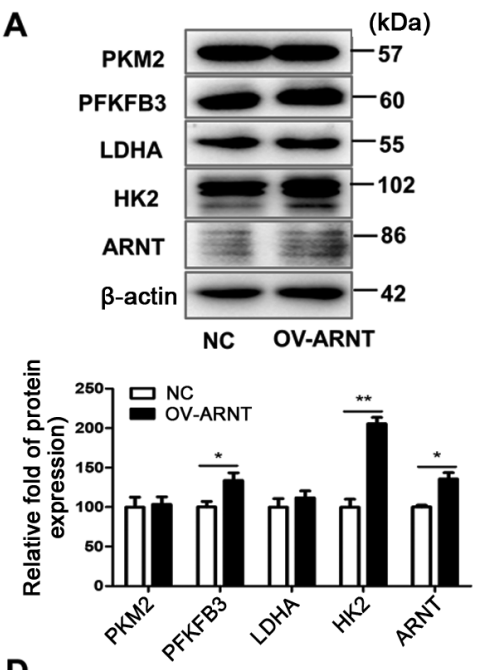

D
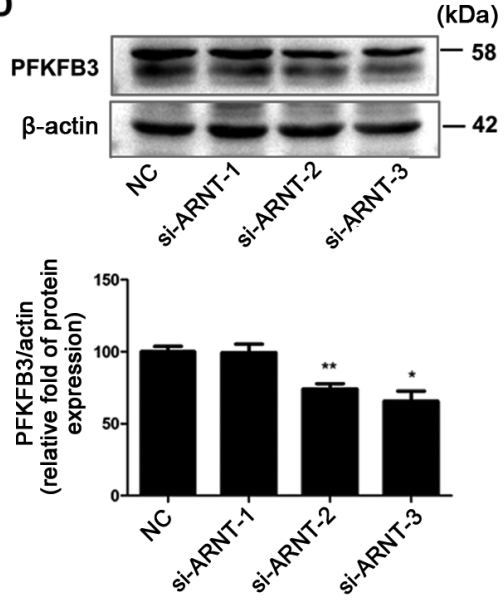

B
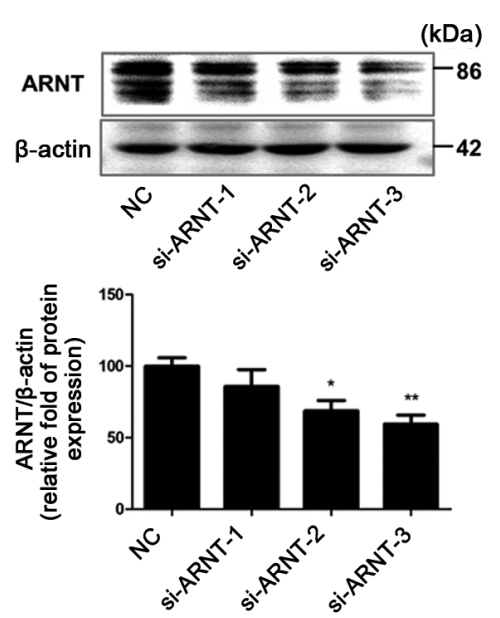

E
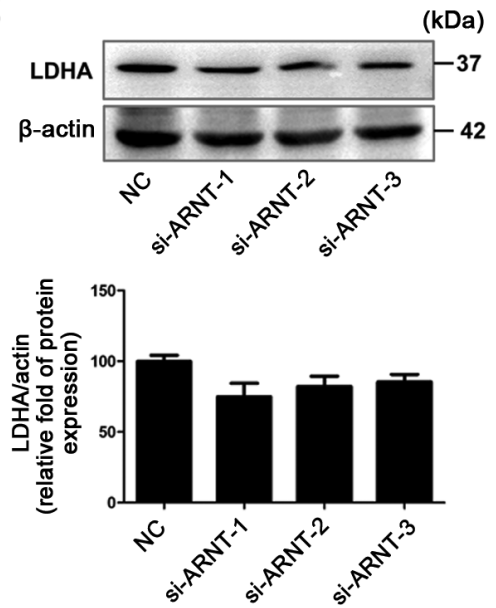

C
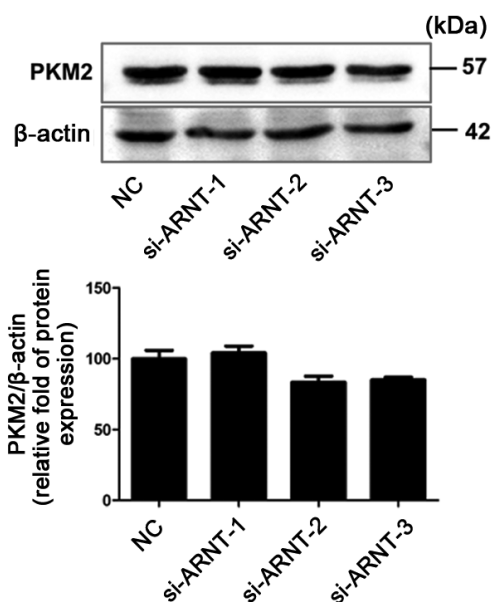

F

HK (kDa)
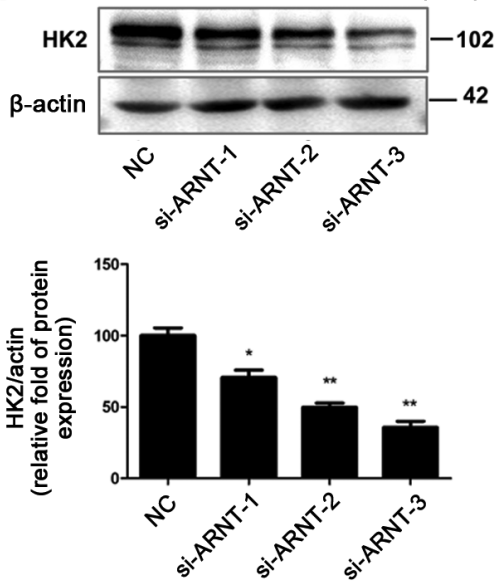

Figure 4. ARNT is associated with the glycolysis signaling pathway in RCC cells. OS-RC-2 cells were transfected with ARNT to overexpress ARNT, while A498 cells were transfected with a lentiviral vector carrying siRNA to silence endogenous ARNT. (A) Protein levels of key glycolysis pathway proteins in OS-RC-2 OV-ARNT cells. Protein levels of (B) ARNT, (C) PKM2, (D) PFKFB3, (E) LDHA and (F) HK2 in A498 si-ARNT cells. $\beta$-actin was used as an internal control. ${ }^{*} \mathrm{P}<0.05$ and ${ }^{* *} \mathrm{P}<0.01$ vs. NC. ARNT, aryl hydrocarbon receptor nuclear translocator; PFKFB3, 6-phosphofructo-2-kinase/fructose-2,6-bisphosphatase 3; LDHA, lactate dehydrogenase; HK2, hexokinase; PFK, phosphofructokinase; PKM2, M2 type acetone kinase.

Effects of ARNT on the glycolysis pathway in RCC cell lines. The effect of ARNT on glycolysis pathway-related proteins was assessed in RCC cell lines. Western blot analysis revealed markedly increased ARNT protein levels in OS-RC-2 OV-ARNT cells and significantly decreased ARNT protein levels in A498-si-ARNT-2/3 cells, compared with the respective NCs (Fig. 4A and B). Overexpression of ARNT in RCC cells led to upregulated HK2 and PFKFB3 expression compared with the NC (Fig. 4A). By contrast, downregulation of ARNT caused significantly decreased protein levels for certain glycolysis pathway members, namely PFKFB3 and HK2, compared with the NC (Fig. 4B-F). These results suggest that ARNT is involved in the regulation of glycolysis.

\section{Discussion}

It has been reported that the HIF signaling pathway consists of three $\alpha$ subunits (HIF- $1 \alpha$, HIF- $2 \alpha$ and HIF- $3 \alpha$ ) and two $\beta$ subunits (ARNT and ARNT2) (22). The major difference between the $\alpha$ and $\beta$ subunits is the type of regulation; the regulation of HIF-1 $\alpha$ is influenced by oxygen tension, whereas ARNT is constitutively and ubiquitously expressed. In contrast to ARNT, ARNT2 is only expressed in the central nervous system, in the kidneys and in breast cancer (11).

In the present study, it was found that ARNT expression was significantly upregulated in patients with ccRCC. The present research on ARNT function is limited, and the regulation of ARNT is still poorly understood and somewhat controversial. Most studies report that ARNT is not regulated by oxygen tension (11). However, Wang et al (23) reported that ARNT mRNA and protein were specifically detected in cells exposed to hypoxia. These findings suggest that ARNT is a hypoxia-inducible protein similar to HIF-1 $\alpha$. Furthermore, Chilov et al (10) demonstrated that the hypoxic inducibility of ARNT occurs in specific cell lines. In the study, ARNT was induced in L929 and Hepal cell lines under hypoxic conditions; however, ARNT levels did not change in human HeLa, Hep3B and LN229 cells when exposed to hypoxic conditions. Vavilala et al (24) and Mandl et al (25) provided additional support for cell line specificity in the hypoxia-dependent regulation of ARNT, suggesting that ARNT is upregulated under hypoxia, for example in human melanoma cells. Previous studies indicate that ARNT is important for normal angiogenesis and glycolysis, and that it may impart anti-apoptotic effects (17). However, its expression and function 
in ccRCC has not been reported. To the best of our knowledge, the present study is the first to demonstrate that ARNT is highly expressed in patients with ccRCC. ARNT expression was also increased in A498 and 786-O RCC cell lines, and ARNT expression was significantly upregulated in kidney cancer according to the Oncomine database, suggesting that the regulation of ARNT may be involved in the development of ccRCC.

Therefore, the function of ARNT in the ccRCC process was studied. Overexpression of ARNT led to a stronger migratory ability and accelerated cell proliferation. According to the Guidelines for the Diagnosis and Treatment of Renal Cancer (26), sunitinib is used as the first-line therapy for RCC; however, National Comprehensive Cancer Network guidelines do not recommend sorafenib as first-line treatment for patients with ccRCC. Since sorafenib has a good tolerance and has shown high efficiency in the Asian population, it is still recommended as a first-line treatment in some renal cancer patients in China. Therefore, it is meaningful to research the mechanism of sorafenib in Chinese ccRCC patients. In the present study, the results also showed that ARNT inhibition reduces accelerated cell proliferation in response to sorafenib treatment, suggesting that targeting ARNT could represent a novel approach to kidney cancer therapy.

Previous studies have indicated that ARNT regulates the transcription of a number of genes involved in glucose metabolism and vascular functions, including phosphoglycerate kinase 1, vascular endothelial growth factor, plasminogen activator inhibitor 1 and erythropoietin (27-30). ARNT may also regulate aldolase in breast carcinomas and in hepatoma cells $(27,28)$. In the present study, ARNT was shown to regulate key enzymes involved in glycolysis. In addition, HK2 and PKM2 were upregulated in RCC cells overexpressing ARNT, suggesting that ARNT contributes to the cancer program through effects on the glycolytic pathway.

Furthermore, it has been reported that mutations of the von Hippel-Lindau (VHL) gene are the main driver events in ccRCC, and loss of VHL products will alter the expression of HIF-1 $\alpha / 2 \alpha$ and their downstream targets (31). As continuously activated HIF forms a pseudo-anoxic state, ccRCC cells are consequently in a pseudo-hypoxic state: HIF-1 $\alpha$ is stabilized and hypoxia inducible genes are upregulated (32). Therefore, the HIF-1 $\alpha$ stabilization also leads to higher ARNT levels as a compensatory mechanism. Future research will be focused on this area.

Overall, the present study indicated that ARNT is involved in regulating glycolysis and cell proliferation in ccRCC. Therefore, ARNT may play an important role in kidney cancer and could represent a new potential therapeutic target for ccRCC.

\section{Acknowledgements}

The authors would like to thank Mr Deqiang Huang (Department of Gastroenterology, Research Institute of Digestive Diseases, The First Affiliated Hospital of Nanchang University, Nanchang, Jiangxi, China) for providing suggestions on the design of the present study.

\section{Funding}

The present study was supported by grants from the National Nature Science Foundation of China (grant no. 31460304) and the Nature Science Foundation of Jiangxi province of China (grant nos. 20181BAB205050, 20192BAB205072 and 20171BCB23086).

\section{Availability of data and materials}

The datasets used and/or analyzed during the present study are available from the corresponding author on reasonable request.

\section{Authors' contributions}

$\mathrm{DH}$ and $\mathrm{XZ}$ designed the experiments. $\mathrm{YZ}$ and $\mathrm{FH}$ performed the experiments. YZ and $\mathrm{CZ}$ analyzed the data. YZ wrote the manuscript. All the authors read and approved the final manuscript.

\section{Ethics approval and consent to participate}

The present study was conducted in accordance with the Declaration of Helsinki. The Ethics Committee of The Second Hospital of Shandong University approved the study. The participants approved the use of clinical samples by providing written informed consent.

\section{Patient consent for publication}

Not applicable.

\section{Competing interests}

The authors declare that they have no competing interests.

\section{References}

1. Epidemiology, pathology, and pathogenesis of renal cell carcinoma. UpToDate 2020.

2. Hsieh JJ, Purdue MP, Signoretti S, Swanton C, Albiges L, Schmidinger M, Heng DY, Larkin J and Ficarra V: Renal cell carcinoma. Nat Rev Dis Primers 3: 17009, 2017.

3. Lopez-Beltran A, Carrasco JC, Cheng L, Scarpelli M, Kirkali Z and Montironi R: 2009 update on the classification of renal epithelial tumors in adults. Int J Urol 16: 432-443, 2009.

4. Vander Heiden MG, Cantley LC and Thompson CB: Understanding the Warburg effect: The metabolic requirements of cell proliferation. Science 324: 1029-1033, 2009.

5. Zheng J: Energy metabolism of cancer: Glycolysis versus oxidative phosphorylation (Review). Oncol Lett 4: 1151-1157, 2012.

6. Favaro E, Lord S, Harris AL and Buffa FM: Gene expression and hypoxia in breast cancer. Genome Med 3: 55, 2011.

7. Li W, Qiu Y, Hao J, Zhao C, Deng X and Shu G: Dauricine upregulates the chemosensitivity of hepatocellular carcinoma cells: Role of repressing glycolysis via miR-199a:HK2/PKM2 modulation. Food Chem Toxicol 121: 156-165, 2018

8. Huang Y, Lin D and Taniguchi CM: Hypoxia inducible factor (HIF) in the tumor microenvironment: Friend or foe? Sci China Life Sci 60: 1114-1124, 2017.

9. McKeown SR: Defining normoxia, physoxia and hypoxia in tumours-implications for treatment response. Br J Radiol 87: 20130676, 2014.

10. Chilov D, Camenisch G, Kvietikova I, Ziegler U, Gassmann M and Wenger RH: Induction and nuclear translocation of hypoxia-inducible factor-1 (HIF-1): Heterodimerization with ARNT is not necessary for nuclear accumulation of HIF-1alpha. J Cell Sci 112: 1203-1212, 1999.

11. Mandl M and Depping R: Hypoxia-inducible aryl hydrocarbon receptor nuclear translocator $(\mathrm{ARNT})(\mathrm{HIF}-1 \beta)$ : Is it a rare exception? Mol Med 20: 215-220, 2014. 
12. Abel $\mathbf{J}$ and Haarmann-Stemmann T: An introduction to the molecular basics of aryl hydrocarbon receptor biology. Biol Chem 391: 1235-1248, 2010.

13. Zagórska A and Dulak J: HIF-1: The knowns and unknowns of hypoxia sensing. Acta Biochim Pol 51: 563-585, 2004.

14. Gunton JE, Kulkarni RN, Yim S, Okada T, Hawthorne WJ, Tseng YH, Roberson RS, Ricordi C, O'Connell PJ, Gonzalez FJ and Kahn CR: Loss of ARNT/HIF1beta mediates altered gene expression and pancreatic-islet dysfunction in human type 2 diabetes. Cell 122: 337-349, 2005.

15. da Silva Xavier G, Rutter J and Rutter GA: Involvement of Per-Arnt-Sim (PAS) kinase in the stimulation of preproinsulin and pancreatic duodenum homeobox 1 gene expression by glucose. Proc Natl Acad Sci USA 101: 8319-8324, 2004.

16. Levisetti MG and Polonsky KS: Diabetic pancreatic beta cells ARNT all they should be. Cell Metab 2: 78-80, 2005.

17. Fernandez-Salguero PM, Ward JM, Sundberg JP and Gonzalez FJ: Lesions of aryl-hydrocarbon receptor-deficient mice. Vet Pathol 34: 605-614, 1997.

18. Huang Q, Sun Y, Ma X, Gao Y, Li X, Niu Y, Zhang X and Chang C: Androgen receptor increases hematogenous metastasis yet decreases lymphatic metastasis of renal cell carcinoma. Nat Commun 8: 918, 2017.

19. Ju L, Zhang X, Deng Y, Han J, Yang J, Chen S, Fang Q, Yang Y and Jia W: Enhanced expression of survivin has distinct roles in adipocyte homeostasis. Cell Death Dis 8: e2533, 2017.

20. Escudier B, Eisen T, Stadler WM, Szczylik C, Oudard S, Siebels M, Negrier S, Chevreau C, Solska E, Desai AA, et al: Sorafenib in advanced clear-cell renal-cell carcinoma. N Engl J Med 356: 125-134, 2007.

21. Wilhelm SM, Carter C, Tang L, Wilkie D, McNabola A, Rong H, Chen C, Zhang X, Vincent P, McHugh M, et al: BAY 43-9006 exhibits broad spectrum oral antitumor activity and targets the $\mathrm{RAF} / \mathrm{MEK} / \mathrm{ERK}$ pathway and receptor tyrosine kinases involved in tumor progression and angiogenesis. Cancer Res 64: 7099-7109, 2004.

22. Rankin EB and Giaccia AJ: The role of hypoxia-inducible factors in tumorigenesis. Cell Death Differ 15: 678-685, 2008.

23. Wang GL, Jiang BH, Rue EA and Semenza GL: Hypoxia-inducible factor 1 is a basic-helix-loop-helix-PAS heterodimer regulated by cellular O2 tension. Proc Natl Acad Sci USA 92: 5510-5514, 1995.
24. Vavilala DT, Ponnaluri VK, Vadlapatla RK, Pal D, Mitra AK and Mukherji M: Honokiol inhibits HIF pathway and hypoxia-induced expression of histone lysine demethylases. Biochem Biophys Res Commun 422: 369-374, 2012

25. MandlM,KapellerB,LieberR and MacfeldaK:Hypoxia-inducible factor-1 $\beta$ (HIF-1 $\beta$ ) is upregulated in a HIF- $1 \alpha$-dependent manner in 518A2 human melanoma cells under hypoxic conditions. Biochem Biophys Res Commun 434: 166-172, 2013.

26. Guidelines for the diagnosis and treatment of renal cancer. National Health Commission of the People's Republic of China 2018.

27. Zelzer E, Levy Y, Kahana C, Shilo BZ, Rubinstein M and Cohen B: Insulin induces transcription of target genes through the hypoxia-inducible factor HIF-1alpha/ARNT. EMBO J 17: 5085-5094, 1998

28. Salceda S, Beck I and Caro J: Absolute requirement of aryl hydrocarbon receptor nuclear translocator protein for gene activation by hypoxia. Arch Biochem Biophys 334: 389-394, 1996.

29. Forsythe JA, Jiang BH, Iyer NV, Agani F, Leung SW, Koos RD and Semenza GL: Activation of vascular endothelial growth factor gene transcription by hypoxia-inducible factor 1 . Mol Cell Biol 16: 4604-4613, 1996.

30. Okino ST, Chichester CH and Whitlock JP Jr: Hypoxia-inducible mammalian gene expression analyzed in vivo at a TATA-driven promoter and at an initiator-driven promoter. J Biol Chem 273: 23837-23843, 1998.

31. Bratslavsky G, Sudarshan S, Neckers L and Linehan WM: Pseudohypoxic pathways in renal cell carcinoma. Clin Cancer Res 13: 4667-4671, 2007.

32. Myszczyszyn A, Czarnecka AM, Matak D, Szymanski L, Lian F, Kornakiewicz A, Bartnik E, Kukwa W, Kieda C and Szczylik C: The role of hypoxia and cancer stem cells in renal cell carcinoma pathogenesis. Stem Cell Rev Rep 11: 919-943, 2015.

This work is licensed under a Creative Commons Attribution-NonCommercial-NoDerivatives 4.0 International (CC BY-NC-ND 4.0) License. 